\title{
Д.В. Павлов
}

Новгородский государственный университет имени Ярослава Мудрого, Великий Новгород, Россия

\section{МЕТОДЫ ИДЕНТИФИКАЦИИ МУЛЬТИПЛИКАТИВНОЙ ПОГРЕШНОСТИ ВОЛОКОННО-ОПТИЧЕСКОГО ГИРОСКОПА}

\begin{abstract}
Представлена модель нелинейностей масштабного коэффициента волоконно-оптического гироскопа (ВОГ) VG910Q. Данная модель ВОГ используется в бесплатформенном инерциальном измерительном модуле, который предназначен для ориентации и навигации. Получены аппроксимации механической и температурной составляющих масштабного коэффициента. Аппроксимирующие полиномы позволяют устранить погрешность масштабного коэффрициента до уровня 0,7 \%, что существенно повышает точность навигационной системы в канале угловой скорости.

Ключевые слова: масштабный коэффициент, волоконно-оптический гироскоп, нелинейность, мультипликативная погрешность, аппроксимация.
\end{abstract}

\section{D.V. Pavlov}

Yaroslav-the-Wise Novgorod State University, Velikii Novgorod, Russian Federation

\section{METHODS OF IDENTIFICATION OF A MULTIPLICATIVE ERROR OF A FIBER-OPTICAL GYROSCOPE}

In this work the model of nonlinearities of scale factor coefficient of a fiber-optical gyroscope (FOG) of VG910Q is presented. The FOG of this model is used in the strapdown inertial measuring module which is intended for orientation and navigation. There have been got the approximations of mechanical and temperature constituents of scaling factor. The approximating polynomials can eliminate an error of scale factor to the level of $0,7 \%$, that significantly increases the accuracy of a navigation system in the channel of angular velocity.

Keywords: scale factor, fiber-optical gyroscope, nonlinearity, multiplicative error, approximation.

Основными проблемами, с которыми сталкиваются разработчики инерциальных навигационных систем, - коррекция погрешностей чувствительных датчиков. Для инерциальных измерителей выделены семь основных составляющих погрешностей [1]. Среди них следует уделить особое внимание мультипликативной погрешности - погрешности масштабного коэффициента. 
В данной работе рассмотрен чувствительный инерциальный элемент - датчик угловой скорости (ДУС) производства «Физоптика». ДУС обычно называют волоконно-оптическим гироскопом, хотя его принцип действия не имеет ничего общего с принципами работы классического гироскопа.

В простейшем случае передаточная функция ВОГ является одномерной:

$$
\operatorname{Uout}_{i}(\omega)=U_{0 i}+k_{i} \cdot \omega+n,
$$

где $U_{0 i}-$ постоянная составляющая при нулевой угловой скорости, В; $k_{i}$ - масштабный коэффициент, который задает наклон прямой $\operatorname{Uout}_{i}(\omega)$, В/град/с; $\operatorname{Uout}_{i}(\omega)$ - функция выходного сигнала ВОГ от угловой скорости; $\omega$ - угловая скорость, град/с; $n$ - случайная составляющая выходного сигнала (белый шум), В; $i$ - значение чувствительных осей ВОГ: $x, y, z$.

В пределах динамического диапазона ВОГ функция (1) является линейной. Однако в области угловых скоростей, приближающихся к границам динамического диапазона, наблюдается отклонение функции от линейности. На границе рабочего диапазона нелинейность масштабного коэффициента является характерным явлением для интерферометрических датчиков [2].

На рис. 1 представлены гармонические изменения дифференцированных показаний стендового датчика угла поворота и угловой скорости ВОГ VG910Q.

Точность измерения датчика угла в данном эксперименте составляет 20 угловых секунд.

По рис. 1 видно, что показания датчиков существенно отличаются на границе рабочего диапазона VG910Q. Относительная погрешность составляет порядка 8-10\%. Расхождение показаний датчиков обусловлено погрешностью масштабного коэффициента.

Нелинейность масштабного коэффициента можно разделить на две составляющие: механическую и температурную [3]. В работе [3] приведено соотношение для масштабного коэффициента

$$
K(T, \omega)=K_{0} \cdot k(T) \cdot k(\omega),
$$

где $K_{0}$ - масштабный коэффициент при комнатной температуре $\left(T_{0}\right)$ в области угловых скоростей до 40 град/с; $T$ - температура в граду- 
сах Цельсия; $k(T)$ и $k(\omega)$ - полиномиальные зависимости масштабного коэффициента от температуры и угловой скорости соответственно.

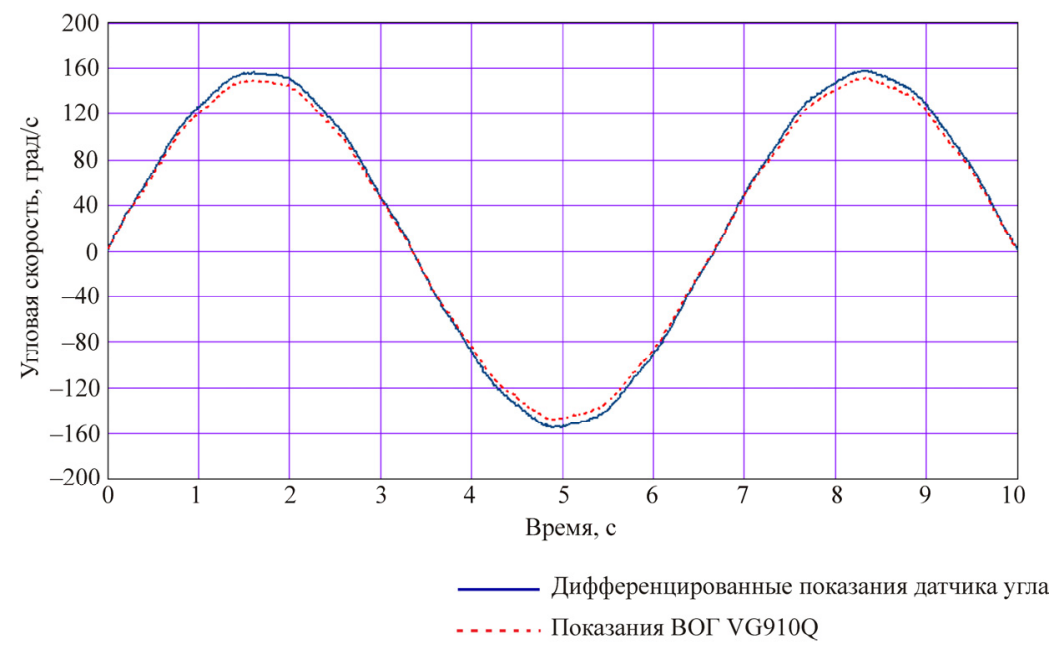

Рис. 1. Гармонические изменения угловой скорости, полученные по показаниям датчиков

Для нахождения функции $k(\omega)$ проводили эксперимент при комнатной температуре (рис. 2).

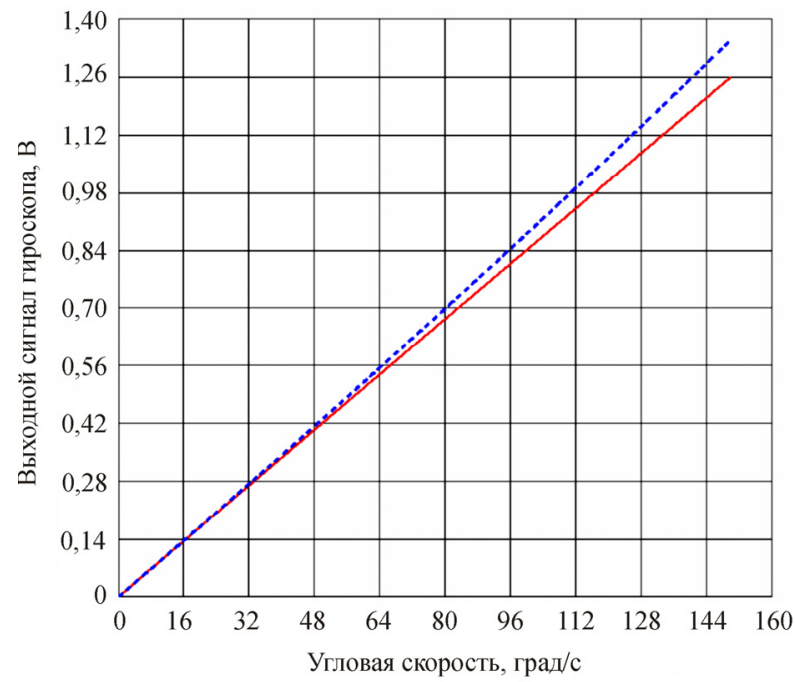

Рис. 2. Зависимость выходного напряжения от угловой скорости: сплошная линия - аппроксимация по паспортным данным VG910Q; пунктирная линия - экспериментальная характеристика 
На стендовом оборудовании увеличивали угловую скорость вращения. Для каждой скорости измеряли выходной сигнал на выходе ВОГ.

По графикам видно, что при скоростях до 60 град/с отклонение выходного сигнала ВОГ от линейного закона не превышает $0,9 \%$. При скоростях выше 60 град/с наблюдается рост нелинейности масштабного коэффициента.

Так как оценка коэффициента $k(\omega)$ не влияет на оценку коэффициента $k(T)$, то их исследования проводятся независимо. Для получения полиномиальной зависимости $k(T)$ модель VG910Q на стенде помещали в климатическую камеру, где устанавливали 20 точек с фиксированной температурой. Для каждой температуры получали зависимость выходного сигнала от угловой скорости. Скорость ограничивали значением 50 град/с, так как при данной скорости механическая составляющая $k(\omega) \approx 1$.

Для минимизации погрешности оценки масштабного коэффициента предложен двухэтапный способ калибровки ВОГ в режиме синусоидального закона изменения угловой скорости с привлечением информации о производной текущего углового положения от стендового датчика угла.

На первом этапе были получены полиномиальные выражения для масштабных коэффициентов $k(T)$ и $k(\omega)$ :

$$
\begin{aligned}
& k^{(1)}(T)=1+1,315 \cdot 10^{-4} \cdot T-2,066 \cdot 10^{-6} \cdot T^{2}, \\
& k^{(1)}(\omega)=1+3,02 \cdot 10^{-6} \cdot \omega^{2}+5,89 \cdot 10^{-11} \cdot \omega^{4} .
\end{aligned}
$$

После первого этапа калибровки на рис. 3 представлены графики синусоидального изменения угловой скорости во времени.

Из рис. 3 видно, что погрешность масштабного коэффициента существенно снизилась и составила уже 3-4 \%.

На втором этапе калибровки масштабного коэффициента получили следующие аппроксимации:

$$
\begin{aligned}
& k^{(2)}(T)=1+8,315 \cdot 10^{-5} \cdot T, \\
& k^{(2)}(\omega)=1+1,77 \cdot 10^{-7} \cdot \omega^{2} .
\end{aligned}
$$

После второго этапа калибровки погрешность масштабного коэффициента снизилась до 0,7 \% (рис. 4). 


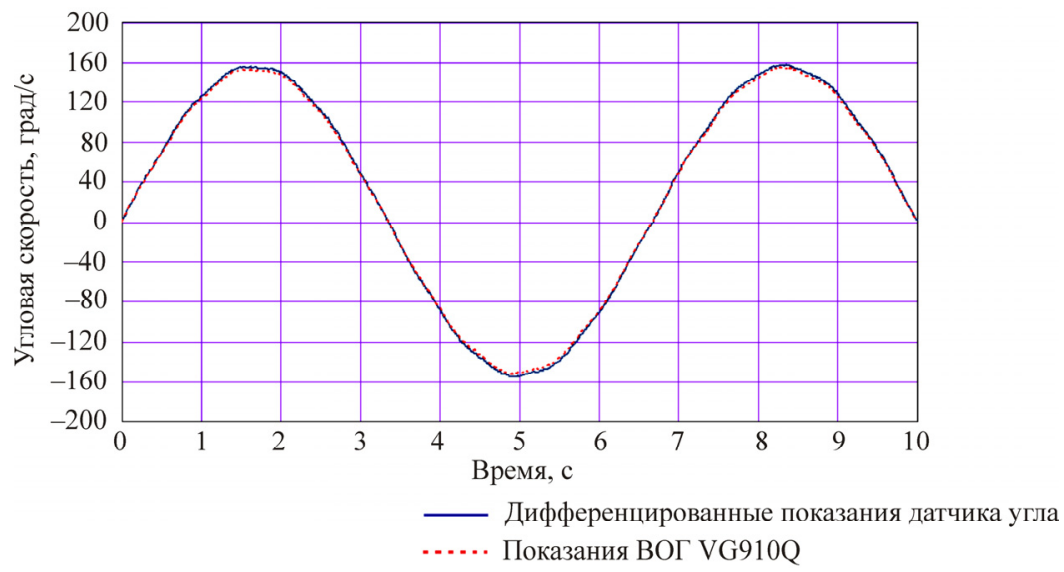

Рис. 3. Сравнение показаний ВОГ и стендового датчика

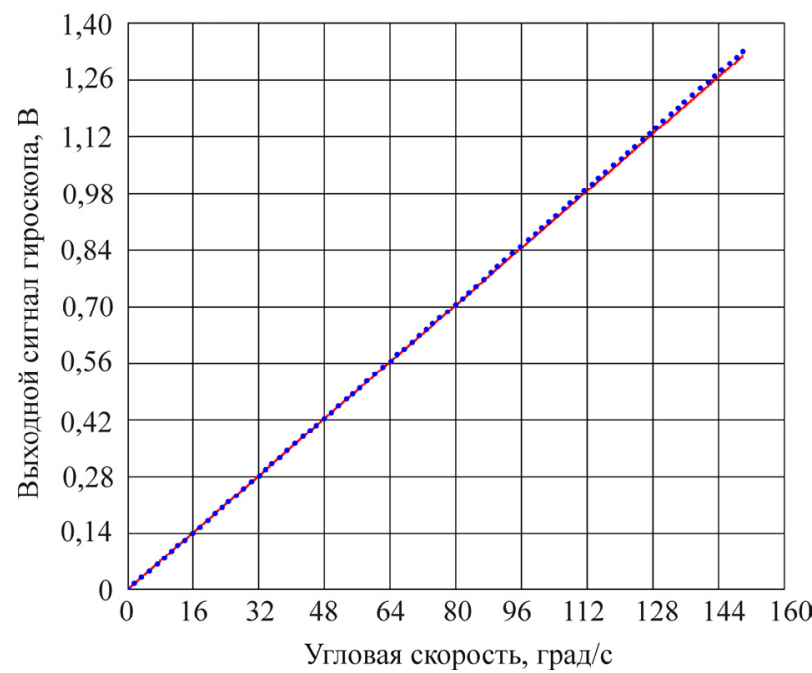

Рис. 4. Зависимость выходного напряжения от угловой скорости: сплошная линия аппроксимация по паспортным данным VG910Q; линия с точками - экспериментальная характеристика после второго этапа калибровки

По рис. 4 видно, что двухэтапные методы калибровки масштабного коэффициента позволили снизить относительную мультипликативную погрешность до уровня 0,7 \%. Данный уровень ошибки позволяет повысить точность систем ориентации и навигации среднего класса точности. 
Bыводы. В работах $[4,5]$ показаны методы коррекции аддитивных погрешностей волоконно-оптического гироскопа. Были получены аппроксимирующие полиномы температурной зависимости дрейфа нуля и СКО шума. В данной работе были получены аппроксимации механической и температурной составляющих масштабного коэффициента. Полученные аппроксимирующие полиномы позволяют устранить погрешность масштабного коэффициента до уровня $0,7 \%$, что существенно повышает точность навигационной системы в канале угловой скорости.

\section{Список литературы}

1. IEEE Std 952-1997 (R2008). IEEE Standard Specification Format Guide and Test Procedure for Single-Axis Interferometric Fiber Optic Gyros. - URL: http://www.control.aau.dk/uav/reports/12gr1052/sources/ books_and_rapports/allan_variance_IEEE.pdf (дата обращения: 10.10.2015).

2. Logozinski V., Listvin V., Solomatin V. Taper twisting for higher FOGs production yield // The 13th International Conference on Optical Fiber Sensors (OFS-13). - Seoul, 2000. - P. 108.

3. Павлов Д.В., Петров М.Н., Лукин К.Г. Разработка динамической модели волоконно-оптического гироскопа // Вестник Новгород. гос. ун-та. Сер.: Техн. науки. - 2012. - № 68. - С.106-109.

4. Поляков Д.В., Лукин К.Г., Петров М.Н. Разработка модели волоконно-оптического гироскопа в среде Simulink // Вестник Новгород. гос. ун-та. - 2012. - № 68.

5. Павлов Д.В. Математическая модель волоконно-оптического гироскопа для статической системы // Прикладная фотоника. - 2014. № 2. - С. 99 .

\section{References}

1. IEEE Std 952-1997 (R2008). IEEE Standard Specification Format Guide and Test Procedure for Single-Axis Interferometric Fiber Optic Gyros, available at: http://www.control.aau.dk/uav/reports/12gr1052/sources/ books_and_rapports/allan_variance_IEEE.pdf (accessed 10 October 2015).

2. Logozinski V., Listvin V., Solomatin V. Taper twisting for higher FOGs production yield. The 13th International Conference on Optical Fiber Sensors (OFS-13). Seul, 2000. 
3. Pavlov D.V., Petrov M.N., Lukin K.G. Razrabotka dinamicheskoi modeli volokonno-opticheskogo giroskopa [Development of a dynamic model of the fiber optic gyroscope]. Vestnik Novgorodskogo gosudarstvennogo universiteta, seriia Tekhnicheskie nauki, 2012, no. 68, pp. 106-109.

4. Poliakov D.V., Lukin K.G., Petrov M.N. Razrabotka modeli volokonno-opticheskogo giroskopa $\mathrm{v}$ srede Simulink [Development of a model of the fiber optic gyroscope in the Simulink medium]. Vestnik Novgorodskogo gosudarstvennogo universiteta, 2012, no. 68.

5. Pavlov D.V. Matematicheskaia model' volokonno-opticheskogo giroskopa dlia staticheskoi sistemy [Mathematical model of a fiber-optic gyroscope for static systems]. Prikladnaia fotonika, 2014, no. 2, p. 99.

Получено 13.11.2015 